\title{
Analysis of the Causes of Optic-Disc Swelling
}

\author{
Jong Jin Jung, Seung-Hee Baek, Ungsoo Samuel Kim \\ Department of Ophthalmology, Kim's Eye Hospital, Konyang University College of Medicine, Seoul, Korea
}

\begin{abstract}
Purpose: To investigate the clinical manifestations and diagnoses of optic disc swelling.
Methods: The medical records of 49 patients who experienced optic disc swelling between March 2008 and June 2009 were retrospectively reviewed. The characteristics of non-arteritic anterior ischemic optic neuropathy (NA-AION) and optic neuritis (ON), which showed optic disc swelling most commonly, were compared.

Results: NA-AION was the most common disorder (34.7\%) that presented with optic disc swelling. ON was identified in 15 patients (30.6\%). Seven out of 49 patients (14.3\%) had intracranially associated diseases, such as papilledema and compressive optic neuropathy. Pseudopapilledema was noted in four patients (8.2\%). Other diseases (e.g., papillophlebitis, neuroretinitis, and diabetic papillopathy) were seen in six patients (12.2\%). Ocular pain was observed more commonly in patients with ON $(p=0.001)$. Patients with ON expected a better visual prognosis than patients with NA-AION $(0.12 \pm 0.32$ vs. $0.49 \pm 0.35, p=0.001)$.

Conclusions: NA-AION and ON should be considered in the differential diagnosis when patients with optic disc swelling present to the neuro-ophthalmology clinic. Detailed history taking and supportive examinations, such as visual field, color-vision and imaging tests, should also be performed as indicated. Regular follow-up of such exams is necessary for the differential diagnosis of these diseases.
\end{abstract}

Key Words: Ischemic optic neuropathy, Korea, Optic disc swelling, Optic neuritis

Optic disc swelling is a pathological condition with a variety of causes. The clinical features associated with unilateral optic disc swelling are demyelinating optic neuritis (ON), non-arteritic anterior ischemic optic neuropathy (NA-AION), compressive optic neuropathy, retinal-vein occlusion, and diabetic papillopathy. Cases with bilateral optic disc swelling are often associated with papilledema, infiltrative optic neuropathy, toxic optic neuropathy, and malignant hypertension [1]. The most common cause of optic disc swelling in Caucasians has been reported to be anterior ischemic optic neuropathy [2]. However, there have been no studies on the common causes and clinical features of optic disc swelling in Asians. Therefore, the purpose of this study was to determine the clinical manifestations and etiology of optic disc edema in Korean patients and to compare the characteristics of NA-AION and ON, the most common causes of optic disc

Received: March 29, 2010 Accepted: September 15, 2010

Corresponding Author: Ungsoo Samuel Kim, MD. Department of Ophthalmology, Kim's Eye Hospital, Konyang University College of Medicine, \#156 Yeongdeungpo-dong 4-ga, Yeongdeungpo-gu, Seoul 150-034, Korea. Tel: 82-2-2639-7777, Fax: 82-2-2677-9214, E-mail: ungsookim@kimeye.com swelling.

\section{Materials and Methods}

We reviewed the clinical records of all patients who presented with optic disc swelling at the initial visit in the neuro-ophthalmology clinic of Kim's Eye Hospital between March 2008 and June 2009. Our study was reviewed and approved by the Kim's Eye Hospital Institutional Review Board.

The optic disc swelling was examined and measured by one neuro-ophthalmologist. Fundus examination and fundus photography were conducted in obvious cases, whereas optical coherence tomography was additionally performed in questionable cases.

The diagnostic criteria were as follows: patients with NA-AION were recruited according to the Ischemic Optic Neuropathy Decompression Trial (IONDT) criteria, and ON was defined by the criteria of Optic Neuritis Treatment Trial (ONTT) [3-4]. The criteria were modified for this study. Patients under the age of 50 were also included in this study, which was different from the IONDT. All ON patients were included regardless of age; moreover, patients treated with steroid pulse therapy were also included.

Papilledema was diagnosed when optic disc edema was 
present with elevated intracranial pressure. Compressive optic neuropathy was confirmed by positive findings on imaging studies. Criteria for the diagnosis of diabetic papillopathy included the presence of diabetes and optic disc edema and the absence of substantial optic-nerve dysfunction, evidence of ocular inflammation or elevated intracranial pressure [5]. The optic disc drusen was defined by ultrasound scanning or computed tomography. Other diseases were diagnosed by their characteristic clinical features.

To compare the difference between NA-AION and ON, the following parameters were included: gender, age, diagnosis, chief complaint, duration of symptoms, presence of ocular pain, and visual acuity at the first and last visits.

For statistical comparison, the Snellen visual acuity was

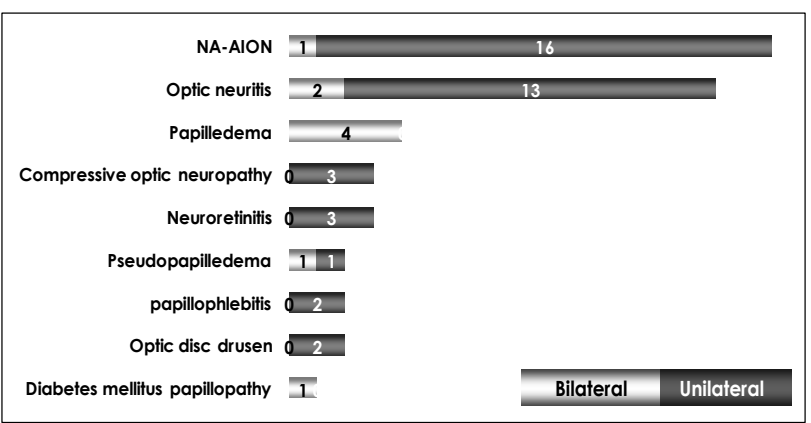

Fig. 1. Diagnoses of patients with optic disc swelling. NA-AION = non-arteritic anterior ischemic optic neuropathy. converted to a logarithm of the minimum angle of resolution ( $\log$ MAR) equivalents. Numerical data were analyzed using the Mann-Whitney test, and categorical variables included the Fisher's exact test. Statistical analyses were performed using the SPSS ver. 15.0 (SPSS Inc., Chicago, IL, USA). A $p$-value of less than 0.05 was considered to be statistically significant.

\section{Results}

Our study included a total of 58 eyes among 49 consecutive patients. The most common disorder was NA-AION (34.7\%). ON was identified in 15 patients (30.6\%), and papilledema was seen in 4 patients. Three patients in each group had compressive optic neuropathy and neuroretinitis. Pseudopapilledema, optic disc drusen and papillophlebitis were diagnosed in two patients in each group. Diabetic papillopathy was present in one patient. Nine patients showed bilateral presentations, four cases had papilledema (100\%), two cases exhibited ON, and one case each had NA-AION, papillophlebitis and diabetes mellitus (DM) papillopathy. (Fig. 1)

Table 1 shows a comparison of characteristics in patients with NA-AION and ON. NA-AION was seen in 17 patients, and 15 patients had ON. The mean age for patients with NA-AION and ON was 53.4 and 29.2 years, respectively. A male predominance was noted in both groups. The chief complaints of the patients in both groups included decreased visual acuity and visual field defects. The duration of symp-

Table 1. Comparison of non-arteritic anterior ischemic optic neuropathy (NA-AION) and optic neuritis (ON) patients

\begin{tabular}{lccc}
\hline & NA-AION $(\mathrm{n}=17)$ & ON $(\mathrm{n}=15)$ & $p$-value \\
\hline Age (yr) & $53.4(42-69)$ & $29.2(9-64)$ & $0.000^{*}$ \\
Sex (M:F) & $11: 6$ & $9: 6$ & $0.636^{\dagger}$ \\
Chief complaint & 11 & 6 & \\
$\quad$ Decreased visual acuity & 6 & 4 & \\
$\quad$ Field defect & $16.12 \pm 17.37$ & $6.4 \pm 7.3$ & $0.009^{*}$ \\
Symptom duration (day) & $1(5.6 \%)$ & $8(53 \%)$ & $0.001^{\dagger}$ \\
Ocular pain & $0.39 \pm 0.41$ & $0.32 \pm 0.37$ & $0.569^{*}$ \\
Initial visual acuity (logMAR) & $0.49 \pm 0.35$ & $0.12 \pm 0.32$ & $0.001^{*}$ \\
Final visual acuity (logMAR) & & & \\
\hline
\end{tabular}

$\log \mathrm{MAR}=$ logarithm of the minimum angle of resolution.

" $p$-value by Mann-Whitney test; ${ }^{\dagger} p$-value by Fisher's exact test.

Table 2. Characteristics of compressive optic neuropathy patients

\begin{tabular}{lccc}
\hline & Case 1 & Case 2 & Case 3 \\
\hline Sex & Female & Male & Female \\
Age & 53 & 59 & 38 \\
Duration of symptom (mon) & 5 & 22 & 2.5 \\
Ocular pain & None & None & None \\
Relative afferent pupillary defect & Positive & Positive & Positive \\
Initial visual acuity & Finger count & 0.3 & Finger count \\
Cause of optic neuritis & Meningioma & Pituitary adenoma & Optic nerve glioma \\
Previous treatment & Steroid pulse & None & Steroid pulse \\
Visual field defect & Total central scotoma & Blind spot enlargement & Total central scotoma \\
\hline
\end{tabular}


toms was significantly greater in the NA-AION group ( $p=$ $0.009)$. Ocular pain was present more frequently in patients with ON $(p=0.001)$. The initial $\log$ MAR visual acuity was $0.39 \pm 0.41$ in the NA-AION group and $0.32 \pm 0.37$ in the $\mathrm{ON}$ group $(p=0.569)$. The visual acuity had a significantly better prognosis in the ON group than in the NA-AION group $(0.12$ \pm 0.32 vs. $0.49 \pm 0.35, p=0.001$ ).

The types of visual field defects varied in this study. The most common visual field defect in the NA-AION group was an inferior altitudinal defect. For the ON patients, altitudinal defect, sectorial scotoma, arcuate scotoma, and blind-spot enlargement presented in similar frequencies.

Compressive optic neuropathy was noted in three patients (Table 2). The causes of compression were sphenoidal meningioma, optic-nerve glioma and pituitary adenoma. All cases presented with a relative, afferent pupillary defect; however, no patient reported ocular pain. Two of the cases had been previously treated with steroid pulse therapy because of misdiagnosis of the $\mathrm{ON}$.

\section{Discussion}

The results of this study showed that the most common disease with optic disc edema was NA-AION; the second most common cause was ON. Patients with retrobulbar ON were not included in this study. Additionally, patients with other disorders that can present with optic disc pallor were also excluded from this study. Pale optic discs that could represent a sequel of $\mathrm{ON}$ were also excluded, so the frequency of $\mathrm{ON}$ might therefore be higher than we found in our study. Although NA-AION has been reported to be the most common cause of acute optic neuropathy in Caucasians 50 years of age and older, optic neuropathy also includes features such as a pale optic disc [6]. Therefore, further information collected by epidemiological studies based on the existence of optic-nerve swelling is necessary.

There was no case of arteritic AION in this study. Some patients with NA-AION were considered to have arteritic AION; however, the diagnosis was not confirmed by blood testing. Kim and Hwang [7] studied Korean patients with AION and reported 2 cases that were C-reactive-protein positive among $25 \mathrm{AION}$ patients. However, these two patients had no general symptoms such as pain (headache) on the temporal side of the involved eye. Therefore, arteritic AION may be a rare disease among Koreans.

NA-AION was diagnosed at an older age in this study. Many patients with NA-AION had other medical conditions (e.g., DM, hypertension, and hypercholesterolemia). These conditions may be associated with the higher rate of NA-AION diagnosed in older patients.

The most common type of visual field defect in NA-AION was an inferior altitudinal defect [8]. The results of this study correlate well with those of previous studies. Other defects, such as arcuate scotoma, central scotoma, blind-spot enlargement, and ring scotoma, were found with similar frequencies in NA-AION and ON.

Ocular pain was present in approximately $90 \%$ of patients in the ONTT [9]. By contrast, about $50 \%$ of patients with ON had ocular pain in this study. Wang et al. [10] studied the etiology and clinical features of 31 Asian patients with ON and reported on the associated ocular discomfort. Several reports have shown a low frequency of ocular pain that is similar to that found in the present study [10-12]. These results might in part be due to ethnic differences.

ON was associated with a better prognosis than NA-AION. The poor prognosis associated with NA-AION may have been due to the irreversible changes of the optic disc that resulted from insufficient blood supply to the optic disc.

Associated intracranial disease was not rare in patients with optic disc swelling (13.4\%; papilledema, 8.2\%; compressive optic neuropathy, 6.1\%) (Table 2). Two of three cases with compressive optic neuropathy had been previously treated with steroid pulse therapy because of misdiagnosed ON. This suggests that unresolved optic disc swelling should be considered in the differential diagnosis of a number of disorders associated with disc edema. Moreover, compressive optic neuropathy usually was not associated with disc swelling. Therefore, careful examination of the central nervous system should be part of the work-up for patients with unusual optic disc swelling.

There are several limitations in this study. The first limitation was the small sample size. We included only 46 patients from one clinic, and all causes of optic disc swelling in Koreans could not be represented by these cases. Secondly, since only the patients who visited the neuro-ophthalmologic clinic initially at the beginning of treatment were included, some patients with diseases (such as diabetic papillopathy) who are often diagnosed in retina clinics might have been overlooked in this study. Moreover, this study design was a retrospective chart review. Some cases were excluded because of insufficient information.

In conclusion, NA-AION and ON should be considered in the diagnosis when patients with optic disc swelling present to neuro-ophthalmology clinics. Detailed history taking, supportive examinations such as visual field, color-vision and imaging tests should also be performed as indicated; regular follow-up of such exams would be necessary for the differential diagnosis of these diseases.

\section{Conflict of Interest}

No potential conflict of interest relevant to this article was reported.

\section{Acknowledgements}

This paper received 2009 Asian Neuro-Ophthalmology Society meeting travel grant. 


\section{References}

1. Van Stavern GP. Optic disc edema. Semin Neurol 2007;27:233-43.

2. Miller NR, Newman NJ, Biousse V, Kerrison JB, editors. Walsh \& Hoyt's clinical neuro-ophthalmology: the essentials. 2nd ed. Philadelphia: Lippincott Williams \& Wilkins; 2008.

3. The ischemic optic neuropathy decompression trial (IONDT): design and methods. Control Clin Trials 1998;19:276-96.

4. Optic Neuritis Study Group. The clinical profile of optic neuritis. Experience of the Optic Neuritis Treatment Trial. Arch Ophthalmol 1991;109:1673-8.

5. Miller NR, Walsh FB, Hoyt WF, editors. Walsh \& Hoyt's clinical neuro-ophthalmology. 6th ed. Philadelphia: Lippincott Williams \& Wilkins; 2005.

6. Kerr NM, Chew SS, Danesh-Meyer HV. Non-arteritic anterior ischaemic optic neuropathy: a review and update. J Clin Neurosci 2009;16:994-1000.

7. Kim DH, Hwang JM. Risk factors for Korean patients with anterior ischemic optic neuropathy. J Korean Ophthalmol Soc
2007;48:1527-31.

8. Hayreh SS, Zimmerman B. Visual field abnormalities in nonarteritic anterior ischemic optic neuropathy: their pattern and prevalence at initial examination. Arch Ophthalmol 2005;123:1554-62.

9. Foroozan R, Buono LM, Savino PJ, Sergott RC. Acute demyelinating optic neuritis. Curr Opin Ophthalmol 2002; 13:375-80.

10. Wang JC, Tow S, Aung T, et al. The presentation, aetiology, management and outcome of optic neuritis in an Asian population. Clin Experiment Ophthalmol 2001;29:312-5.

11. Wakakura M, Minei-Higa R, Oono S, et al. Baseline features of idiopathic optic neuritis as determined by a multicenter treatment trial in Japan. Optic Neuritis Treatment Trial Multicenter Cooperative Research Group (ONMRG). Jpn J Ophthalmol 1999;43:127-32.

12. Lin YC, Yen MY, Hsu WM, et al. Low conversion rate to multiple sclerosis in idiopathic optic neuritis patients in Taiwan. Jpn J Ophthalmol 2006;50:170-5. 